\title{
HPV detection and measurement of HPV-16, telomerase, and survivin transcripts in colposcopy clinic patients
}

\author{
S Lanham, A Herbert, P Watt
}

\begin{abstract}
Aims-To determine whether the detection of high risk human papillomavirus (HPV) types is more predictive for high grade CIN than the current cervical smear test, and whether the production and measurement of HPV type 16 (HPV16) and cellular survivin and telomerase transcripts can be used to discriminate between cervical HPV infections that self cure and those that induce high grade lesions.

Methods-Three hundred and fifty four cervical smear samples from women attending the colposcopy clinic were tested by the polymerase chain reaction (PCR) for the presence of HPV. Transcripts for HPV-16 E6, E6`I, E6^II, E7, and L1 as well as cellular survivin, telomerase RNA component, and telomerase reverse transcriptase were measured using fluorogenic probe (Taqman) assays.

Results-Referral smear grades of severe or moderate showed greater positive predictive values for CIN $2 / 3$ than did the detection of high or moderate risk HPV types. HPV-16 transcripts from E6, E6^I, E6^II, and E7 showed high predictive values for CIN 2/3, but low sensitivity. The telomerase RNA component was detected in $\mathbf{5 3}$ of $\mathbf{5 7}$ samples and telomerase reverse transcriptase was only detected in one sample, whereas survivin transcripts were detected in $40 \%$ of samples.

Conclusions-The detection of HPV-16 or cellular survivin or telomerase transcripts did not accurately predict the grade of CIN in the samples. The detection of HPV risk types correlated well with the grade of CIN; however, the referral grade smear was the most accurate predictor of the severity of the lesion. Of the 35 different HPV types detected, 18 are not included in the HPV hybrid capture II commercial test kit. The use of such kits would have missed HPV infection in $4.3 \%$ of clinic patients with CIN 2/3 lesions and 15.4\% with CIN 0/1.

(F Clin Pathol 2001;54:304-308)
\end{abstract}

Keywords: human papillomavirus type 16; survivin; telomerase; CIN; transcripts; Taqman

Cervical cancer is the second most common cancer in women, with around 500000 new cases each year, and accounting for 200000 deaths worldwide. In the UK, with the national screening programme deaths from cervical cancer fell from around 2000 in 1980 to 1369 in 1994. Estimates suggest that between 5\% and $30 \%$ of women with cervical abnormalities are missed in a single smear test. ${ }^{1}$ Given that women are only screened every three to five years, undetected cervical abnormalities could develop into cancer in the interval between tests. The elimination of cervical cancer requires improved screening; key options are semi-automated preparation and screening of smears, in addition to testing for potentially oncogenic human papillomavirus (HPV) infection.

A commercially available HPV hybrid capture detection kit (Hybrid Capture II; Digene Corporation, Gaithersburg, USA) separates testing into low risk (HPV types 6, 11, 42, 43, and 44) and intermediate/high risk (HPV types $16,18,31,33,35,39,45,51,52,56,58,59$, and 68) HPV types. One objective of our present study was to establish the proportion of women with cervical smear abnormalities, warranting referral for colposcopy, who were infected with HPV types not included in the commercial test panel and whether infection with any of the excluded HPV types was associated with severe abnormalities (CIN grades $2 / 3$ ). A universal screening programme for cervical HPV infection will detect very large numbers of positive women, around $20 \%$ of the sexually active population. In an important study, Ho and colleagues ${ }^{2}$ showed the mean duration of cervical HPV infection to be eight months, and that by 24 months after the initial infection only $9 \%$ of women continued to be infected. Repeated smear testing of those women who spontaneously self cure would have important social and financial consequences. A possible way of discriminating between cervical HPV infections that self cure and those that induce high grade lesions is to determine the pattern of HPV gene transcription. The proposition is that the genes associated with the HPV replication cycle will be transcribed in self healing infections, whereas transcription from the E6 and E7 genes will be upregulated in high grade lesions. HPV-16 infections were chosen for study; this virus accounted for $45.4 \%$ of all HPV infections detected in our colposcopy clinic patients and had a positive predictive value of $73.0 \%$ for CIN 2/3. In addition, transcripts from host cell genes survivin and telomerase, implicated in many cancers, were analysed.

\section{Methods}

PATIENTS

Full ethical approval was given by the south and west local research ethics committee for 
the collection of cervical samples from women attending a colposcopy clinic in the Wessex region of southern England. Women were asked to give written consent to take part in our study.

\section{SAMPLES}

Cervical specimens for molecular analysis were taken during colposcopic examination using a combination of plastic spatula and cytobrush. The CIN grade of the specimen was the highest grade detected in a biopsy taken immediately after the polymerase chain reaction (PCR) sample.

\section{DNA EXTRACTION}

Cell samples were collected into $2.5 \mathrm{ml}$ RPMI medium containing $10 \%$ fetal calf serum (FCS) and $0.05 \%$ sodium azide. Cells were pelleted from $0.5 \mathrm{ml}$ aliquots centrifuged at $60000 \times g$ for 30 minutes. The DNA was extracted with alkali lysis by resuspending the cells in $200 \mu \mathrm{l} 2 \mathrm{M}$ ammonium hydroxide (Applied Biosystems, Warrington, Cheshire, UK). The sealed tubes were placed in a waterbath at $90^{\circ} \mathrm{C}\left( \pm 1^{\circ} \mathrm{C}\right)$ for 10 minutes to denature the virus, then the tubes were opened and reheated for a further 70 minutes to evaporate the ammonia. Aliquots from each specimen were stored at $-80{ }^{\circ} \mathrm{C}$ until used for PCR.

RNA EXTRACTION

Cell samples were collected into $2.5 \mathrm{ml}$ RNAlater solution (Ambion, Austin, Texas, USA). A $0.5 \mathrm{ml}$ aliquot of the solution was mixed with $0.5 \mathrm{ml}$ phosphate buffered saline (PBS) and centrifuged at $60000 \times g$ for five

Table 1 Primers and probes used in fuorogenic probe assays

\begin{tabular}{|c|c|}
\hline Primer name & Sequence $5^{\prime} \rightarrow 3^{\prime}$ \\
\hline E6For & CAAACCGTTGTGTGATTTGTTAATTA \\
\hline E6Rev & GCTTTTTGTCCAGATGTCTTTGC \\
\hline E6Probe & TGTATTAACTGTCAAAAGCCACTGTGTCCTGAAGAA \\
\hline $\mathrm{E} 66^{\star} \mathrm{I}$ & TACTGCGACGTGAGGTGTATTAAC \\
\hline E6*II & TACTGCGACGTGAGATCATCA \\
\hline E6 $6^{\star}$ Common & CAGTTGTCTCTGGTTGCAAATC \\
\hline E6^Probe & TATCTCCATGCATGATTACAGCTGGGTTTCTCTAC \\
\hline E7For & ACCGGACAGAGCCCATTACA \\
\hline E7Rev & GCCCATTAACAGGTCTTCCAAA \\
\hline E7Probe & ATTGTAACCTTTTGTTGCAAGTGTGACTCTACGCTT \\
\hline L1For & CGTTATGACATACATACATTCTATGAATTCC \\
\hline L1Rev & TGGGTTACAAACCTATAAGTATCTTCTAGTGT \\
\hline L1Probe & CTGGAATTTTGGTCTACAACCTCCCCCAG \\
\hline GAPDHFor & GGGAAGGTGAAGGTCGGAGT \\
\hline GAPDHRev & TGGAAGATGGTGATGGGATTTC \\
\hline GAPDHProbe & ATTGACCTCAACTACATGGTTTACATGTTCCAATATGA \\
\hline hTERTFor & CGGAAGAGTGTCTGGAGCAA \\
\hline hTERTRev & CGACGTAGTCCATGTTCACAATC \\
\hline hTERTProbe & CTTCAAGTGCTGTCTGATTCCAATGCTTTGC \\
\hline hTRFor & GGTGGTGGCCATTTTTTGTC \\
\hline hTRRev & CTAGAATGAACGGTGGAAGGC \\
\hline hTRProbe & CGCGCTGTTTTTCTCGCTGACTTTC \\
\hline SurvFor & GCGCTTTCCTTTCTGTCAAGA \\
\hline SurvRev & GCACGGCGCACTTTCTT \\
\hline SurvProbe & ССTCAAATTCTTTCTTCTTATTGTTGGTTTCCTTTGCC \\
\hline
\end{tabular}

The table shows the primer and probe sequences used for the detection of human papillomavirus (HPV) transcripts and cellular telomerase reverse transcriptase (hTERT), telomerase RNA component (hTR), survivin, and glyceraldehyde-3-phosphate dehydrogenase $(\mathrm{GAPDH})$ transcripts. minutes. The cell pellet was lysed with $1 \mathrm{ml}$ Trizol reagent (Life Technologies, Paisley, UK). RNA extraction was carried out according to the manufacturer's instructions. The pellet was vacuum dried for 30 seconds and the RNA dissolved in ultra high quality water by heating at $55^{\circ} \mathrm{C}$ for 10 minutes. Any contaminating DNA was removed by incubating with $1 \mathrm{U}$ of amplification grade DNAse I (Life Technologies) at room temperature for 15 minutes. The enzyme was denatured at $65^{\circ} \mathrm{C}$ for 10 minutes and then reverse transcription was performed. A sample was stored at $-20^{\circ} \mathrm{C}$ as a control to test for DNA contamination.

REVERSE TRANSCRIPTION

The RNA was mixed with 1.5 pmoles of each of primers: 5'-CGTTGCTGCTGCAGGAT-3', 5'GACCACGTCTGCAGGAT-3', and 5'-GCCA CCTAG AACTGCAGGAT-3' (HPV-16 transcripts with nucleotide 880 splice), 5'GTTGCTGCTCACGTCG C-3' and 5'-GA CCACGTCTCACGTCGC-3' (HPV-16 transcripts with nucleotide 226 splice), 5'-GC CCATTGTACCATCTGTGATAAT-3' (transcripts containing the HPV-16 E1 region), L1Rev, GAPDHRev, hTERTRev, hTRRev and SurvRev (table 1), then incubated at $70^{\circ} \mathrm{C}$ for 10 minutes, allowed to cool and the reverse transcription (RT) reaction carried out using 200 units Superscript reverse transcriptase (Life Technologies), according to the manufacturer's instructions. The cDNA solutions were then stored at $-20{ }^{\circ} \mathrm{C}$ until use.

PCR REACTIONS

HPVs were detected using three consensus primers-GP5+/GP6+, ${ }^{3}$ MY09/MY11, ${ }^{4}$ and $\mathrm{CPI} / \mathrm{CPIIG}^{5}$ - as well as specific primers for HPV-16 (forward primer: 5'-GTCAAAAGCC ACTGTGTCCT-3', reverse primer 5'-CCAT CCATTACATCCCGAC-3' producing a $499 \mathrm{bp}$ product), and HPV-18 (forward primer: 5'-ACAATCCTCCATTTTGCTGT G-3', reverse primer: 5'-ATAAACTATGTC TGCACAGCTTA-3' producing a $384 \mathrm{bp}$ product). Human globin gene primers (forward: 5'-GAAGAGCCAAGGACAGGTAC-3' and reverse: 5'-CAACTTCATCCACGTTCA CC-3') gave a 268 bp product if human DNA was present in the sample. All the PCR reactions consisted of $2 \mathrm{mM} \mathrm{MgCl}$ ( $2.5 \mathrm{mM}$ for HPV-18 specific PCR), PCR buffer (Promega, Southampton, UK), deoxynucleotide triphosphates (Promega) each at $300 \mu \mathrm{M}$, primers at $1 \mathrm{mM}$, and $1.5 \mathrm{U}$ of Taq DNA polymerase (Promega). A volume of $5 \mu \mathrm{l}$ of extracted DNA ( 10-100 ng) was used in a total PCR volume of $25 \mu \mathrm{l}$. The PCR was performed on an MJ Research PTC-225 machine for a total of 40 cycles. PCR conditions for HPV-16 and HPV-18 were $94^{\circ} \mathrm{C}$ for 30 seconds, $60^{\circ} \mathrm{C}\left(62^{\circ} \mathrm{C}\right.$ for $\mathrm{HPV}-18$ and $55^{\circ} \mathrm{C}$ for globin) for 50 seconds, and $72^{\circ} \mathrm{C}$ for 20 seconds. The HPV types detected with consensus primers were identified by sequencing of the PCR product and aligning to HPV sequences at Genbank using the FastA program $^{6}$. 


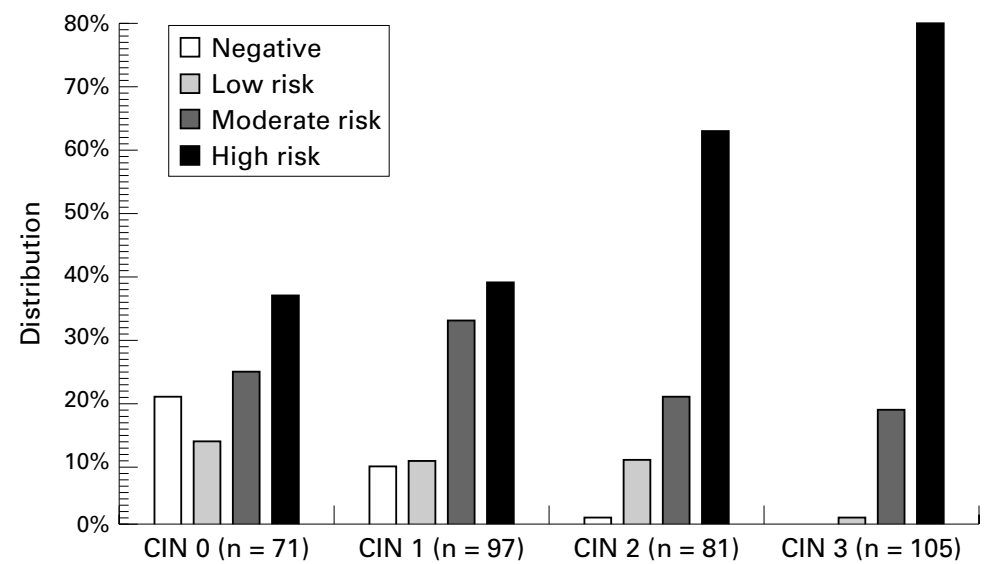

Figure 1 The distribution of human papillomavirus risk type with grade of CIN.

TRANSCRIPT MEASUREMENT IN HPV-16 POSITIVE SAMPLES

Probes were labelled with 6-FAM at the $5^{\prime}$ end and TAMRA at the $3^{\prime}$ end. The reaction mixture for the fluorogenic probe assays used the Taqman core reagent kit (Applied Biosystems) with $3.5 \mathrm{mM} \mathrm{MgCl}_{2}$, forward and reverse primers at $900 \mathrm{nM}$, and probe at $200 \mathrm{nM}$ (table 1). A $5 \mu \mathrm{l}$ aliquot of the cDNA mixture was used in a total volume of $25 \mu \mathrm{l}$ using the manufacturer's cycling conditions on an Applied Biosystems Prism 7700 sequence detector.

\section{Results}

CERVICAL HPV INFECTION IN COLPOSCOPY CLINIC PATIENTS

Cervical samples from 354 women attending the colposcopy clinic were tested by PCR for the presence of HPV DNA using the consensus primers GP5F/GP6+, CPI/CPIIG, and MY09/ MY11. The GP primers detected HPV in 43 CIN 0 samples, 72 with CIN 1, 75 with CIN 2, and 97 samples with CIN 3. CP primers were used on samples that were negative using the GP primers and detected a further three HPV positive samples with CIN 0, five samples with CIN 1, two with CIN 2, and five with CIN 3. The MY primers detected HPV in an additional seven samples with CIN 0, six with CIN 1 , and one with CIN 3.The remaining negative samples were screened by specific PCR for the E7 gene of HPV-16, which detected a further four HPV positive samples with CIN 1, three samples with CIN 2, and two samples with CIN 3. PCR detecting the long control region of HPV-18 detected a further three HPV positive samples with CIN 0. A single patient with
CIN 2 repeatedly tested negative with all HPV primers. All 26 samples that gave negative PCR results with the HPV primers tested positive for the human globin gene by PCR, confirming that none of the samples was inhibitory for PCR or lacked DNA. The 328 samples (92.7\%) containing detectable HPV DNA were typed by direct sequencing of the PCR product. Figure 1 shows the results. Of the 35 different HPV types detected in our clinic patients 18 types are not included in either the HPV intermediate/high risk or the low risk commercial test kit panels. The use of such kits would have missed HPV infection in $4.3 \%$ of clinic patients with CIN 2/3 lesions and $15.4 \%$ with CIN 0/1.

Referral smear grades were strongly correlated with the colposcopy clinic findings, with severe grade having a positive predictive value (PPV) of $89.3 \%$ for CIN 2/3, moderate grade $60.5 \%$, and persistent mild or borderline nuclear abnormalities having a PPV of $25.6 \%$. Even within the context of women referred for colposcopy, the detection of different HPV risk types predicted biopsy findings less accurately, with high risk HPV having a PPV for CIN 2/3 of $67.5 \%$, moderate risk a PPV of $42.0 \%$, and low risk HPV types a PPV of $32.3 \%$.

ONCOGENIC TRANSCRIPTS IN WOMEN INFECTED WITH HPV-16

The protein products of the E6 and E7 genes of potentially oncogenic HPV types are believed to play a crucial role in the development of cervical cancer. Fluorogenic probe assays were developed to measure the HPV-16 transcripts from the E6 gene, truncated E6*I and E6 ${ }^{\star}$ II transcripts, plus the E7 and L1 genes. In addition, transcripts from host cell genes survivin and telomerase, implicated in many cancers, were analysed. To validate the assays, cDNA from two tumour cell lines containing HPV-16 (CaSki and $\mathrm{SiHa}$ ) and tumour tissue from a highly malignant and a slow growing HPV-16 containing cervical cancer was prepared. Transcripts of the E6 and E7 oncogenes were detected in all tumours (table 2), but the transcript copy number in each cell was extremely low in the slow growing cancer. Similarly, transcripts of survivin were 100 times lower in this tumour and telomerase reverse transcriptase could not be detected.

To determine whether the presence of oncogene transcripts would accurately differentiate between low grade $(\mathrm{CIN} 0 / 1)$ and high grade (CIN 2/3) lesions cDNA was prepared from 57

Table 2 Transcript values detected in cell lines and cervical tumours

\begin{tabular}{|c|c|c|c|c|c|c|c|c|c|}
\hline \multirow[b]{2}{*}{ Cells } & \multicolumn{5}{|c|}{$H P V-16$ transcript } & \multicolumn{4}{|c|}{ Cellular transcript } \\
\hline & E6 & $E 6^{\star} I$ & $E 6^{\star} I I$ & E7 & $L 1$ & $G A P D H$ & hTERT & $h T R$ & Survivin \\
\hline CaSki & $2 \times 10^{-1}$ & Not detected & Not detected & 6.00 & $5 \times 10^{-3}$ & 7.00 & $6 \times 10^{-5}$ & 1.00 & $2 \times 10^{-1}$ \\
\hline $\mathrm{SiHa}$ & 1.00 & 1.00 & $4 \times 10^{-2}$ & 9.00 & $2 \times 10^{-3}$ & 126.00 & $3 \times 10^{-3}$ & 2.00 & 3.00 \\
\hline Tumour 1 & 2.00 & 2.00 & $8 \times 10^{-2}$ & 42.00 & $1 \times 10^{-4}$ & 18.00 & $8 \times 10^{-4}$ & 4.00 & $5 \times 10^{-1}$ \\
\hline Tumour 2 & $8 \times 10^{-3}$ & $1 \times 10^{-3}$ & Not detected & $8 \times 10^{-2}$ & $5 \times 10^{-4}$ & $7 \times 10^{-1}$ & Not detected & $6 \times 10^{-2}$ & $3 \times 10^{-3}$ \\
\hline
\end{tabular}

DNA and RNA were extracted from cervical cancer cell lines containing HPV-16 (CaSki and SiHa) and biopsies from two patients with a cervical tumour containing HPV-16: a fast growing tumour from a 34 year old women (tumour 1) and a slow growing tumour from a women of 78 (tumour 2).

Transcript measurements were performed by fluorogenic probe assay and are presented as copy number/cell. The average HPV-16 genome copy number/cell was 815 for CaSki cells, 3 for SiHa cells, $6 \times 10^{-1}$ for the fast growing tumour, and 11 for the slow growing tumour

GAPDH, glyceraldehyde-3-phosphate dehydrogenase; hTERT, human telomerase reverse transcriptase; hTR, human telomerase RNA component. 
Table 3 Proportion of CIN samples with transcripts

\begin{tabular}{|c|c|c|c|c|c|}
\hline \multirow[b]{2}{*}{ Transcript } & \multicolumn{3}{|c|}{ No. positive samples (\% positive) } & \multicolumn{2}{|c|}{$\begin{array}{l}\text { Positive predictive value } \\
\text { and specificity for CIN } 2 / 3\end{array}$} \\
\hline & $\begin{array}{l}C I N 0 / 1 \\
(n=17)\end{array}$ & $\begin{array}{l}C I N 2 / 3 \\
(n=40)\end{array}$ & p Value & $P P V$ & Specificity \\
\hline E6 & $6.00(35.3)$ & $29.00(72.5)$ & 0.01 & 82.90 & 64.70 \\
\hline E6*I & $2.00(11.8)$ & $17.00(42.5)$ & 0.01 & 89.50 & 88.20 \\
\hline E6*II & $0.00(0)$ & $10.00(25)$ & 0.04 & 100.00 & 100.00 \\
\hline E7 & $5.00(29.4)$ & $24.00(60)$ & 0.04 & 82.80 & 70.60 \\
\hline $\mathrm{L} 1$ & $6.00(35.3)$ & $23.00(57.5)$ & 0.13 & 79.30 & 64.70 \\
\hline hTERT & $1.00(5.9)$ & $0.00(0)$ & 0.12 & 0.00 & 94.10 \\
\hline hTR & $14.00(82.4)$ & $37.00(92.5)$ & 0.25 & 72.50 & 17.60 \\
\hline Survivin & $8.00(47.1)$ & $15.00(37.5)$ & 0.50 & 65.20 & 52.90 \\
\hline
\end{tabular}

The table shows the proportion of colposcopy samples containing HPV-16 that contained detectable amounts of the transcripts shown. The number in each group (n) is shown. HPV-16 transcripts are E6, E6 ${ }^{\star}$ I, E6 ${ }^{\star}$ II, E7, and L1. E6 and E7 encode the full length functional protein, whereas E6*I and E6 $6^{\star} \mathrm{II}$ encode truncated E6 proteins whose functions are uncertain.

Cellular transcripts are telomerase reverse transcriptase (hTERT), telomerase RNA component (hTR), and survivin.

p Values were calculated using $\chi^{2}$ analysis.

Also shown are the subsequent positive predictive value (PPV) and specificity in determining CIN $2 / 3$ for each transcript.

Glyceraldehyde-3-phosphate dehydrogenase (GAPDH) transcripts were detected in all samples.

colposcopy clinic patients infected with HPV16. Table 3 presents the assay results. GAPDH transcripts were detected in all samples. Transcripts from E6 and the truncated products, E6 ${ }^{\star} \mathrm{I}$ and $\mathrm{E} 6{ }^{\star} \mathrm{II}$, were more commonly detected in CIN 2/3 than CIN 0/1 ( $p=0.01$ using $c 2$ analysis). Overall, detection of E6 transcripts had a PPV of over $82 \%$ for CIN $2 / 3$, but a sensitivity of less than $73 \%$. Although the E6 ${ }^{\star}$ II transcript was not detected in CIN $0 / 1$, detection had a sensitivity of only $25 \%$ for CIN $2 / 3$. Comparable results were obtained with transcripts from the E7 gene, which had a PPV of $82.8 \%$ for CIN 2/3, but could not be detected in $40 \%$ of patients with this grade of lesion.

Monitoring transcripts of host cell genes involved in malignancy provided no conclusive results. The telomerase RNA component was detected in all but four samples and telomerase reverse transcriptase was only detected in one sample, which was CIN 0/1. Survivin transcripts were detected in $40 \%$ of samples regardless of the grade of cervical lesion.

\section{Discussion}

The detection of a high risk HPV type was $67.5 \%$ predictive of CIN 2/3, whereas the detection of a moderate risk type was $42 \%$ predictive, and a low risk HPV type $32.3 \%$ predictive of a high grade lesion. Clearly, women with smear abnormalities with low risk type HPV infection must be referred for colposcopy. Approximately $7.5 \%$ of women attending for colposcopy were referred with persistent mild or moderate smear abnormalities, but their smears did not contain detectable HPV DNA. No significant abnormality was detected in these patients, implying they need not have been referred for colposcopy. However, the use of commercially available HPV test kits to exclude women from colposcopy would present real problems because HPV hybrid capture detection kits divide HPV types into two test groups, namely: low risk (HPV types 6, $11,42,43$, and 44) and intermediate/high risk (HPV types 16, 18, 31, 33, 35, 39, 45, 51, 52, 56, 58, 59, and 68) HPV types. In those patients referred for colposcopy in Southampton the use of a combination of intermediate/ high and low risk HPV tests would have missed HPV infection in $4.3 \%$ in CIN $2 / 3$ and $15.4 \%$ in CIN 0/1. One key problem is that 18 of the 35 genital HPV types (six low risk and 12 undetermined risk types) detected in the Southampton group are not included in the commercial test panels and the oncogenic potential of some types such as han831 (detected in three samples with CIN 2 and four with CIN 1) remains to be established.

HPV-16 cervical infection was chosen to study the production of viral transcripts in the different grades of CIN to determine whether HPV oncogenic transcription could be used to predict the outcome of a high risk HPV infection. In the patients with high grade cervical lesions (CIN 2/3), there was a larger proportion of samples expressing transcripts for HPV-16 E6, E6 ${ }^{\star} I, E 6^{\star} I I$, and E7 compared with those patients with low grade lesions (CIN $0 / 1$ ). Only a longitudinal study could establish whether women with CIN 0/1 samples expressing these transcripts are likely to progress to high grade CIN.

Studies on cervical cancers ${ }^{7}$ demonstrated that telomerase RNA component (hTR) was present in all tumours and normal cervical cells, whereas telomerase reverse transcriptase (hTERT) was only present in tumours. These data fit the results of our study except that low level expression of hTERT was found in one patient presenting with low grade CIN. Hence, the detection of these transcripts does not appear to be useful in determining the likely outcome of HPV-16 infection.

Survivin is expressed in the G2/M phase of the cell cycle and its synthesis is rapidly downregulated after cell cycle arrest. ${ }^{8}$ Thus, survivin would not be present in exfoliated epithelial cells, but is present in cervical cancers and other tumours of epithelial cell origin. ${ }^{9}$ The detection of survivin mRNA, therefore, would be expected in cervical cancers and when cervical cells are induced into continuous replication by HPV-16 infection. The low level of expression of survivin in the CIN samples was anticipated because only a minority of cells in the sample would be HPV infected. Survivin transcripts were detected in approximately $40 \%$ of women infected with HPV-16, regardless of the severity of the cervical lesion.

Expression of the survivin protein allows the cell to replicate continuously by a different mechanism to the HPV-16 E6 protein, which binds the cellular $\mathrm{p} 53$ protein. Coexpression of survivin might enable the virus to induce cellular replication with lower concentrations of E6 protein. One interesting possibility is that enhanced cellular proliferation in cells expressing survivin might require lower concentrations of E6 protein. The mean amount of HPV-16 E6 transcripts in the samples was seven times lower in CIN 2/3 samples coexpressing survivin transcripts ( $\mathrm{p}=0.04$ using the Student's $t$ test), whereas the mean expression values of E7 $(p=0.9)$ and L1 $(p=0.1)$ were not different in these samples.

Finally, the smear result correlated well with the colposcopy biopsy result; for patients with a 
referral smear of borderline nuclear abnormalities (BNA), $78.3 \%$ of samples subsequently had low grade lesions (CIN 0/1); similarly, $73.5 \%$ of patients with a referral smear result of mild dyskaryosis had CIN $0 / 1$. In contrast, $60.5 \%$ of patients with moderate dyskaryosis at referral had high grade lesions (CIN 2/3) at colposcopy, and for patients with referral smears with severe dyskaryosis, $89.3 \%$ of samples subsequently had CIN $2 / 3$. These results indicate the high predictive value of determining dyskaryosis using the current cervical screening methodology.

This work was funded by a grant from the Medical Research Council.

1 Fricker J. Cervical-cancer screening comes of age-or does it? Lancet 1997;350:1010.
2 Ho GYF, Bierman R, Beardsley L, et al. Natural history of cervicovaginal papillomavirus infection in young women. $N$ Engl f Med 1998;338:423-8.

3 de Roda Husman AM, Walboomers JMM, van den Brule AJC, et al. The use of general primers GP5 and GP6 elongated at their $3^{\prime}$ ends with adjacent highly conserved sequences improves human papillomavirus detection by PCR. F Gen Virol 1995;76:1057-62.

4 Manos MM, Ting Y, Wright DK, et al. The use of polymerase chain reaction amplification for the detection of genital human papillomavirus. Cancer Cells 1989;7:209-14.

5 Tieben LM, ter Schegget J, Minaar RP, et al. Detection of Tieben LM, ter Schegget J, Minaar RP, et al. Detection of
cutaneous and genital HPV types in clinical samples by PCR cutaneous and genital HPV types in clinical samples by PCR
using consensus primers. F Virol Methods 1993;42:265-80.

6 Program manual for the Wisconsin package, version 8, August 1994, Genetics Computer Group, 575 Science Drive, Madison, Wisconsin, USA 53711.

7 Takakura M, Kyo S, Kanaya T, et al. Expression of human telomerase subunits and correlation with telomerase activity in cervical cancer. Cancer Res 1998;58:1558-61.

$8 \mathrm{Li} \mathrm{F}$, Ambrosini G, Chu EY, et al. Control of apoptosis and mitotic spindle checkpoint by survivin. Nature 1998;396: $580-4$.

9 Ambrosini G, Adida C, Altieri DC. A novel anti-apoptosis gene, survivin, expressed in cancer and lymphoma. Nat Med 1997;3:917-21.

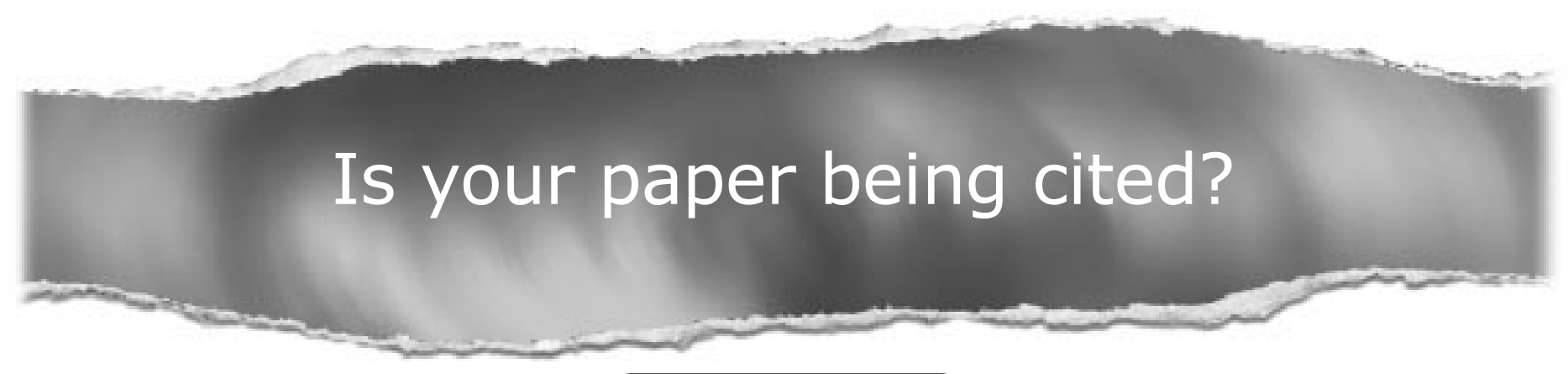

\section{CiteTrack service}

CiteTrack will alert you by email whenever new content in the Journal of Clinical Pathology or a participating journal is published that matches criteria you want to track

Topics: Tell CiteTrack which words or subjects to watch for in new content Authors: Be alerted whenever key authors you are following publish a new paper Articles: Know whenever a paper of interest to you is referenced by another paper

\section{www.jclinpath.com}

